\title{
COMPARISON OF PERILIMBAL SUCTION CUP ANALYSIS AND TONOGRAPHY IN THE DIAGNOSIS OF GLAUCOMA*
}

\author{
BY \\ P. A. MACFAUL \\ Department of Experimental Ophthalmology, Institute of Ophthalmology, London
}

IN the past few years there has been increasing interest in the use of the pressure cup technique for the investigation of aqueous humour dynamics.

Rosengren (1956) demonstrated a rise of intra-ocular pressure when a positive pressure was applied to the sclera overlying Schlemm's canal. He suggested that this rise of pressure was due to continued formation and accumulation of aqueous humour, the escape of which was prevented by occlusion of the outflow channels. The scleral pressure was exerted by a plastic cup with a flange fitting over the limbal region and held in position by vacuum. It has been shown that outflow of aqueous can be prevented by the application of a negative pressure of $50 \mathrm{~mm}$. $\mathrm{Hg}$ in the cup, thereby creating a pressure of $28 \mathrm{~mm}$. $\mathrm{Hg}$ on the sclera, provided that the intra-ocular pressure does not exceed $30 \mathrm{~mm}$. Hg (Langham, 1963).

From the induced rise of pressure several investigators have estimated the rate of aqueous formation. Such values have tended to be lower than with other methods. This may have been due to escape of aqueous as the intra-ocular pressure builds up, or to suppression of aqueous formation with rising intra-ocular pressure. It is also possible that the suction cup flange may interfere with blood flow in the anterior segment and consequently affect aqueous formation.

Considerable interest attaches to the features of the pressure decay curve following removal of the cup. From this and from the induced rise of pressure, Ericson (1958) claimed that it is possible to calculate the rate of aqueous formation, the coefficient of facility of outflow, rate of aqueous drainage, and the outflow venous pressure. Assuming that the intra-ocular pressure will decay at a rate dependent on the outflow facility of the eye, Galin, Baras, and Nano (1962) introduced the "percentage pressure decay" (P.P.D.) as an estimate of outflow resistance.

The purpose of this paper is to report on a comparison between outflow values obtained by the suction cup technique and by tonography. In particular, it was intended to discover:

(1) Whether the intra-ocular pressure fell exponentially to the level existing before application of the suction cup.

(2) Whether there was any variation between the values of $\mathrm{C}$ calculated for different segments of the decay curve.

\footnotetext{
* Received for publication February 19, 1965.
} 
(3) Whether a correlation existed between values of $\mathrm{C}$ derived from the suction cup results and those obtained by tonography.

(4) Whether the percentage pressure decay gave a reliable indication of outflow resistance.

(5) Whether one test was more efficient than the other in differentiating between glaucomatous and non-glaucomatous eyes.

\section{Material and Methods}

A total of 50 eyes was studied in a consecutive series of 26 patients referred for investigation of known or suspected glaucoma to the Glaucoma Clinic at the Institute of Ophthalmology, London.

In 24 patients both eyes were studied. In two others, each with unilateral central retinal vein occlusion, only the affected eye was examined. The series also included 6 eyes with high myopia.

Each eye was examined, using the suction cup technique and tonography, but the two tests were done on separate days.

\section{Suction Cup}

The instrument used was similar to that described by Galin, Baras, and McLean (1963). The device consists of a plastic funnel-shaped cup of diameter $12 \mathrm{~mm}$. with a 5-mm. flange of curvature $15 \mathrm{~mm}$. This is placed on the anaesthetized eye with the flange overlying the limbal region. It is held in place by suction created with a simple aneroid device connected to the cup by a plastic tube.

The procedure was the same in each case and was explained to the patient. Surface anaesthesia was obtained with amethocaine drops 2 per cent. Pre-suction and all subsequent pressure measurements were made with the Goldmann applanation tonometer. The patient was then placed on a couch with the back at $45^{\circ}$ and the head supported in a more or less vertical position. The suction cups were placed in position on each eye and a negative pressure of $50 \mathrm{~mm}$. $\mathrm{Hg}$ was applied. Blanching of the limbal vessels, movement of the cup with the globe, steaming of the internal surface of the cup, and resistance to gentle traction provided evidence of a good fit. After 15 minutes' suction the cups were removed and the intra-ocular pressure recorded as soon as possible (least delay, 1.5 minutes). Thereafter the pressure was measured at 4- or 5-minute intervals for a period between 15 and 50 minutes, the duration of which depended on the course of the pressure decay.

\section{Tonography}

On a subsequent occasion tonography was done on the supine patient using the Schwarzer electro-tonometer and recording unit. All the tonograms were recorded by the same observer.

\section{Calculations}

By plotting pressure against time from the pressure decay curve obtained with the suction cup method, data were obtained with which the percentage pressure decay and coefficient of facility of outflow $\mathbf{C}_{\mathbf{s}}$ were calculated.

The percentage pressure decay is the percentage of the induced rise of pressure which is lost in the first fifteen minutes after removal of the suction cup. It is easily calculated. Owing to the delay in recording the first post-suction pressure the intra-ocular pressure immediately after removal of the cup is never known exactly. It was deduced by drawing a best-fit curve through the points of the decay curve and extrapolating back to zero time. 
Alternatively, in some cases a straight line could be obtained by plotting the decay curve logarithmically.

From the pressure decay curve the coefficient of facility of outflow $\mathrm{C}_{\mathrm{s}}$ was calculated using the formula:

$$
\begin{aligned}
& \mathrm{C}_{\mathrm{s}}=\frac{\Delta \mathrm{V}}{\left(\mathrm{P}_{\mathrm{tav}}-\mathrm{P}_{\mathrm{ps}}\right) \mathrm{Xt}} \\
& \text { where } \Delta V=\frac{1}{K}\left(\log P_{1}-\log P_{2}\right)
\end{aligned}
$$

$P_{p s}$ was the "pre-suction" intra-ocular pressure, $P_{1}$ and $P_{2}$ being any two pressures on the curve separated by an interval of time $t$ minutes.

In each case $C_{s}$ was calculated using (a) the average value $K=0.0215$ and (b) the value for $\mathrm{K}$ obtained from the applanation pressure and initial tonographic reading. This calculation was made for three successive periods of 4 minutes on the decay curve.

\section{Course of Pressure Decay Curve}

\section{Results}

The first question to be answered was whether the pressure fell exponentially to the level existing before application of the suction cup. This was assessed in two ways:

(1) By simple inspection of the curve to see if the pressure fell below the pre-suction level.

(2) By plotting $\log \left(\mathbf{P}_{t}-\mathbf{P}_{\mathrm{ps}}\right)$ against time.

In 17 eyes the pressure fell exponentially and in a further 22 eyes the decay of pressure was probably exponential. In 11 eyes the pressure decay was not exponential. In 9 out of the 11 eyes in the last group there was a rapid return to a level below the original pre-suction level.

Figs 1,2, and 3 show examples of typical decay curves obtained in this series.

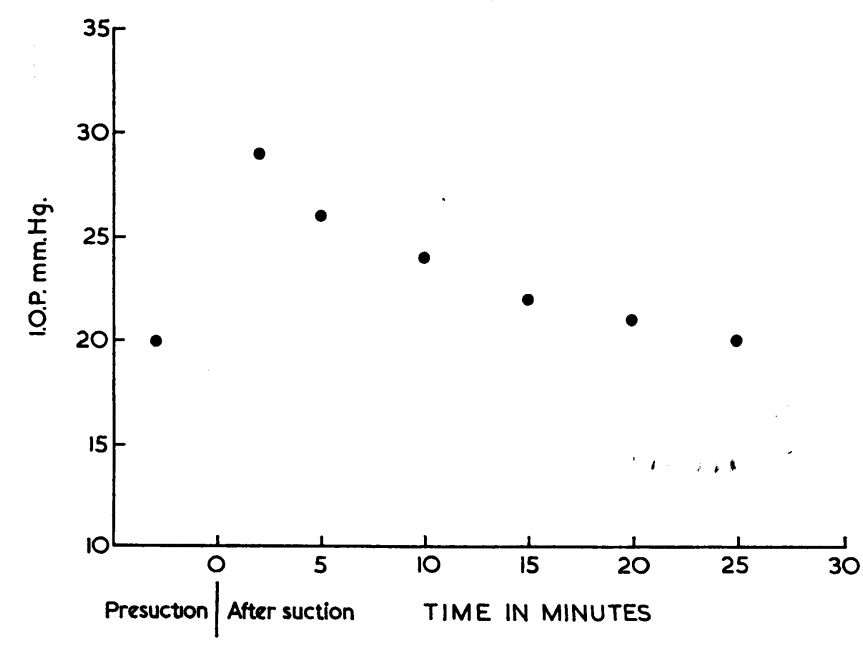

Fig. 1.-Pressure decay curve showing the exponential fall towards the pre-suction level in a glaucomatous eye. 


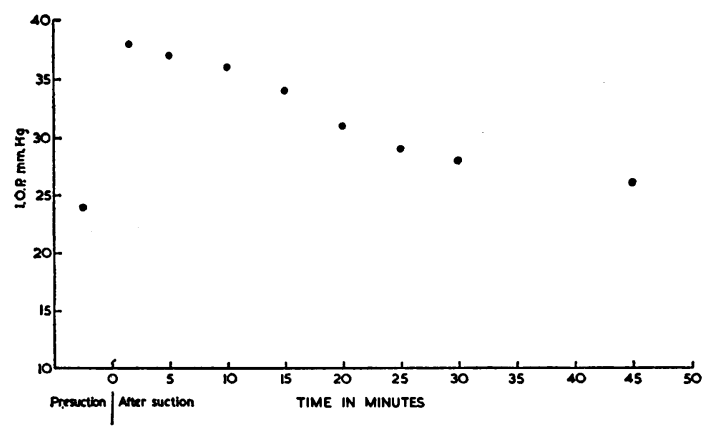

FIG. 2.-Pressure decay curve in a glaucomatous eye showing an initial plateau followed by an exponential fall.

Fig. 1 shows the exponential fall towards the pre-suction level in a glaucomatous eye; Fig. 2 an initial plateau followed by an exponential fall in a glaucomatous eye; and Fig. 3 a rapid fall to a level below the pre-suction level in a normal eye.

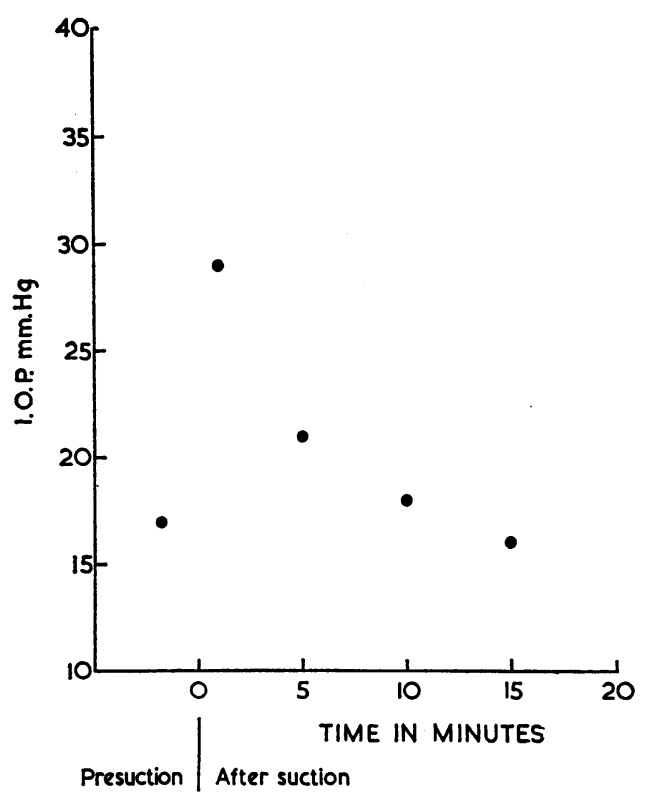

FIG. 3.-Pressure decay curve in an eye with normal outflow showing a rapid fall to a level below the pre-suction level.

\section{Variation in $\mathbf{C}$ during Decay Curve}

The mean values of $\mathrm{C}_{\mathrm{s}}$ for the 50 eyes calculated for different segments of the decay curve are shown in Table $\mathrm{I}$.

The results show that the $C$ values are higher when calculated from the latter part of the curve and these differences with time were found to be statistically significant. From a consideration of tests on individual eyes, there was a clear tendency towards a higher value of $\mathrm{C}_{\mathrm{s}}$ in the 8-12-minute period as compared with the 0-4-minute period. There was no significant difference in the $C_{s}$ values when calculated with and without the correction for ocular rigidity. Hereafter, only the uncorrected values of $C_{s}$ and $\mathrm{C}_{\mathrm{t}}$ will be considered.

TABLE I

Mean Values of $C_{s}$ for 50 Eyes

\begin{tabular}{c|c|c|c|c|c|c}
\hline & \multicolumn{2}{|c|}{ Uncorrected for Ocular Rigidity } & \multicolumn{2}{c}{ Corrected for Ocular Rigidity } \\
\cline { 2 - 6 } $\begin{array}{c}\text { Time period (minutes) } \\
\text { on decay curve }\end{array}$ & $0-4$ & $4-8$ & $8-12$ & $0-4$ & $4-8$ & $8-12$ \\
\hline C. Mean of 50 eyes & 0.09 & 0.11 & 0.15 & 0.09 & 0.11 & 0.15 \\
\hline Standard deviation & 0.045 & 0.075 & 0.116 & 0.06 & 0.08 & 0.12 \\
\hline
\end{tabular}




\section{Correlation between Suction Cup and Tonography}

In order to assess the correlation between suction cup outflows and tonographic outflows, values for $\mathrm{C}$ obtained from three successive 4-minute periods of the suction cup decay curve were plotted against the values of $\mathrm{C}$ from the first four minutes of tonography. According to Armaly (1964) the 4-minute outflow is a good representative of outflow facility prevailing during each individual minute of tonography. Fig. 4 shows the relationship between uncorrected suction cup $C$ values and uncorrected tonographic $\mathrm{C}$ values for the period $0-4$ minutes. Although there is a significant correlation for the $0-4$ minutes segment, the $C_{s}$ values are generally lower than the tonographic values. Table II shows the correlation between tonographic $\mathrm{C}$ and suction cup $\mathrm{C}$ calculated from various segments of the pressure decay curve.

TABLE II

Correlation between Tonographic C and Suction Cup C

\begin{tabular}{c|c|c|c}
\hline Segment of Decay Curve & $0-4 \mathrm{~min}$. & $4-8 \mathrm{~min}$. & $8-12 \mathrm{~min}$. \\
\hline $\begin{array}{c}\text { Correlation } \\
\text { coefficient }\end{array}$ & $\begin{array}{c}0.34 \\
0.05>\mathbf{P}>0.01\end{array}$ & $\begin{array}{c}0.29 \\
0.05>\mathrm{P}>0.01\end{array}$ & $\begin{array}{c}0.19 \\
\mathbf{P}>0.05\end{array}$ \\
\hline
\end{tabular}

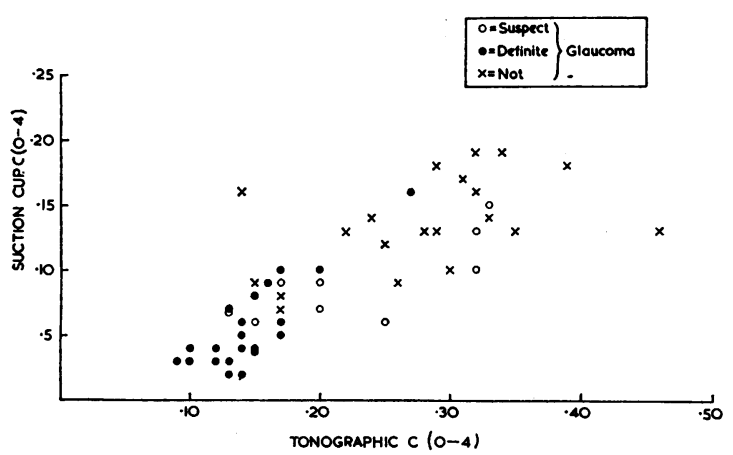

ig. 4.- Scattergram showing the relationship between ncorrected suction cup $C$ values and uncorrected tonoraphic $C$ values for the period $0-4$ minutes.

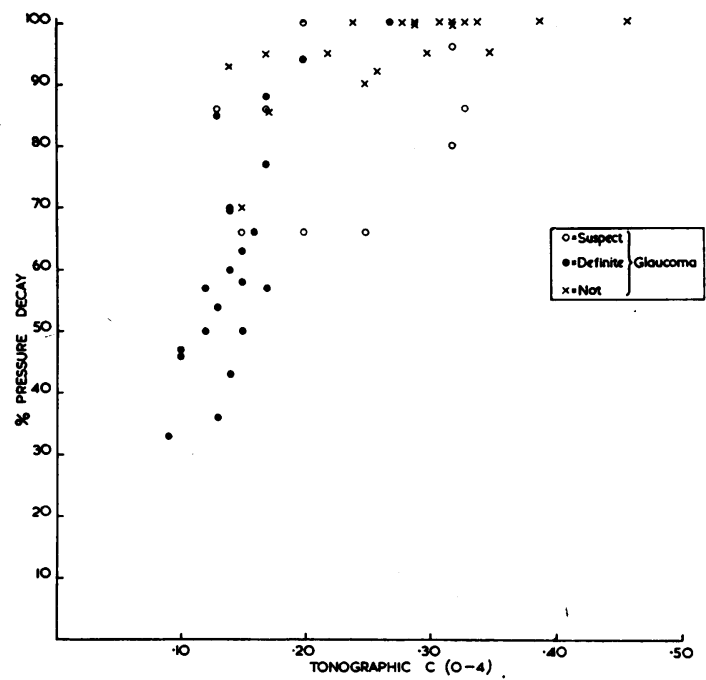

FIG. 5.-Scattergram showing the relationship between percentage pressure decay and tonographic outflow values.

\section{Percentage Pressure Decay Curve}

To assess whether the percentage pressure decay gives a reliable indication of outflow resistance the values of percentage pressure decay were plotted against the tonographic outflow values as shown in Fig. 5. Statistical analysis gave a correlation coefficient of $r=0.43(0.01>P>00)$. 


\section{Diagnostic Value of Suction Cup}

In order to assess the value of the suction cup data in separating glaucomatous eyes from non-glaucomatous eyes the case histories were reviewed and divided into three groups on the basis of disc appearance, open angles, presence of field defects, and raised tensions $(>21 \mathrm{~mm}$. $\mathrm{Hg}$ on the Goldmann applanation tonometer). Definite glaucoma included those cases with cupped discs, field defects, and raised tensions; glaucoma suspect are those cases with suspicious discs and tensions between 21 and 24 on applanation; not glaucoma are those cases with normal discs, absence of field defects, and normal tensions. Tonographic data were excluded from this assessment. The number of eyes in each group was as follows: definite glaucoma, 21 eyes; suspect glaucoma, 9 eyes; not glaucoma, 20 eyes, giving a total of 50 eyes.

Table III shows the number of eyes with tonographic $\mathrm{C}<0 \cdot 15$ and with a percentage pressure decay of $<70$ per cent. An uncorrected tonographic $C$ value of $0 \cdot 15$ or under was taken as probably, and under $0 \cdot 12$ as definitely, pathological. Galin, Baras, Nano, and Cavero (1963) stated that a tonographic value of 0.18 or greater correlated well with a percentage pressure decay of 70 per cent. or more, whereas a value of 0.15 correlated well with a percentage pressure decay of 70 per cent. or less.

TABLE III

Tonographic C Values and Percentage Pressure Decay

\begin{tabular}{l|c|c|c}
\hline & $\begin{array}{c}\text { No. of Eyes with } \\
\text { Tonographic C } \\
<0 \cdot 15\end{array}$ & $\begin{array}{c}\text { No. of Eyes with } \\
\text { P.P.D. } \\
<70 \text { per cent. }\end{array}$ & $\begin{array}{c}\text { Total No. } \\
\text { of } \\
\text { Eyes }\end{array}$ \\
\hline Definite glaucoma & 16 & 16 & 21 \\
\hline Glaucoma suspect & 2 & 3 & 9 \\
\hline Not glaucoma & 2 & 1 & 20 \\
\hline
\end{tabular}

\section{Discussion}

Since Ericson (1958) introduced the suction cup technique several reports have appeared concerning its use on patients. Reporting on a series of more than 400 eyes Langham and Maumenee (1964) emphasized the absence of side-effects. Although it appears to be without harmful effect certain difficulties of a practical nature have been encountered in this series.

Failure of suction due to poor fit occurred several times. In some cases this could probably have been prevented by the use of cups with different diameters.

Dislodgement attributable to lack of flexibility of the plastic tube may have been aggravated by the vertical position of the head in this series. This was to some extent overcome by the use of a head-band which supported the tubes at right angles to the globe, but this arrangement was found to be cumbersome.

With surface anaesthesia of the globe the patient did not feel the cup, but many experienced a drawing sensation. In some cases, especially those with small palpebral fissures, contact between the cup and the sensitive internal surface of the eyelids caused discomfort and squeezing which led to dislodgement of the cup. 
No corneal complications were observed, but many eyes were congested and hyperaemic after the period of suction.

The suction cups were in place for 15 minutes. This is rather a long time for many people to keep still, and most preferred the much shorter period needed for tonography. The suction cup test might be less uncomfortable if done in the supine position with the tubes coming from above, but the vertical position was necessary because of the decision to use applanation measurements throughout. A portable applanation tonometer would avoid this difficulty and would also allow more rapid measurement of intra-ocular pressure at the end of suction. In cases with impaired outflow the prolonged decay time resulted in the test taking between 1 and $1 \frac{1}{2}$ hours compared with an average 30-45 minutes for tonography.

One value of this technique is that it can be used to investigate the outflow of patients on whom tonography is impossible. Thus it may be of value in patients with nystagmus or poor central fixation, provided that reliable applanation tensions can be obtained, or when the patient cannot lie flat because of arthritis or heart failure. A few patients expressed a definite preference for tonography.

It is important to consider whether the suction cup has any effect on the production of aqueous humour, either as a result of the induced rise of intra-ocular pressure or perhaps from local interference with blood flow. The flange of the cup covers an area about $5 \mathrm{~mm}$. wide around the limbus when fitting well. This area includes the territory of the anterior ciliary artery system, the distribution of which has been described by Ashton and Smith (1953). Arising from the arteries to each rectus muscle the anterior ciliary arteries pass forward from the insertion towards the limbus. Three groups of branches arise: small episcleral vessels joining the limbal and anterior conjunctival plexuses, small intrascleral vessels passing deeply to terminate around the canal of Schlemm, and a group of larger arteries which perforate the sclera near the limbus and contribute to the formation of the major arterial circle of the iris from which the ciliary body is supplied. Blanching of the limbal vessels referred to earlier as one of the signs associated with a well-fitting cup, suggests occlusion of these vessels, but whether such occlusion, if indeed it occurs, causes any significant reduction in blood flow to the ciliary body is not known.

Even if the cup does not interfere with inflow there is a strong possibility that it continues to interfere with outflow after its removal. A period of relative ischaemia could lead to oedema in the tissues of the drainage area and perhaps contribute to delayed re-opening of the outflow channels. Calculations of the $C_{s}$ value for the first 4-minute segment gave significantly lower values than when calculated from subsequent segments of the decay curve. This was particularly evident in the glaucomatous eyes.

Two patterns emerge from a study of the decay curves. In some eyes with normal outflows and no clinical evidence of glaucoma the pressure fell exponentially towards the pre-suction level, but in other normal eyes a pressure below the pre-suction level was reached in 10-20 minutes. The group with impaired outflow showed features similar to those described by Chandler (1964), e.g., prolonged decay time up to 50 minutes or longer, and an initial plateau.

The initial plateau (see Fig. 2) is important. Reference has been made earlier to the difficulty in arriving at the intra-ocular pressure at the time of release of suction 
because of the inevitable delay in moving the patient to the applanation tonometer. Increasing values for $\mathrm{C}_{\mathrm{s}}$ could mean that the outflow channels were reopening to their true capacity. Langham (1962) presented tonographic support for the view that the outflow channels do not reopen to their pre-existing capacity immediately after removal of the cups, an effect which in the present series appears to be most marked in eyes with impaired outflow. Low values of $\mathrm{C}_{s}$ in the first four minutes may therefore be due to a combination of two factors: difficulty in measuring the first post-suction pressure, or incomplete opening of the outflow channels immediately after the period of suction.

Reference to Fig. 4 and Table II shows that although there is a significant correlation between suction cup $\mathrm{C}$ and tonographic $\mathrm{C}$ the suction cup values are generally lower than the tonographic values. There are many sources of error in obtaining a value for facility of outflow in tonography and probably also with the suction cup, some of which have already been discussed. The difference may simply be a reflection of the systematic errors of the two methods; for instance, tonography may over-estimate $\mathrm{C}$ and suction cup under-estimate $\mathrm{C}$. Tonographic outflows were calculated with the correction for elevation of episcleral venous pressure as is the usual practice. This calculation was not applied to the suction cup results since we have no knowledge of the induced elevation of episcleral venous pressure. The effect of occlusion of the episcleral veins and subsequent engorgement which was noted in some eyes increased the difficulty in this respect. If neither test predominantly over-estimates or under-estimates $C$ then there is the possibility that the suction cup does not completely occlude the outflow channels.

After the period of suction the intra-ocular pressure should decay at a rate dependent on the outflow facility of the eye. Therefore measuring the percentage return to pre-suction level should provide some indication of resistance to outflow. The relationship between percentage pressure decay and tonographic $\mathrm{C}$ is shown in Fig. 5 . From this it can be seen that 12 out of 21 glaucomatous eyes had a percentage pressure decay less than the lowest percentage pressure decay in the non-glaucoma group. From Fig. 4 it can be seen that 10 out of 21 glaucomatous eyes had a $C_{t}$ lower than the lowest $C_{t}$ in the non-glaucoma group and that 12 glaucomatous eyes had a $C_{s}$ less than the lowest $C_{s}$ in the non-glaucoma group. The two tests therefore are approximately equal in power of differentiation between glaucomatous and normal eyes.

If the suction cup technique does interfere with the outflow channels it is an important factor, because it means that the technique disturbs the actual parameter which it is supposed to be measuring. Although it appears that the calculation of $\mathrm{C}$ from the suction cup is open to question, the qualitative features of the decay curve do help in separating the eyes with impaired outflow from those with normal outflow, and this is probably the greatest value of this test in the diagnosis of glaucoma.

\section{Summary}

Outflow values obtained by the perilimbal suction cup technique and tonography are compared in a series of 50 eyes referred for investigation of known or suspected glaucoma. 
The suction cup test was done with the head upright and applanation measurements were used to follow the decay of pressure.

A correlation was found between tonographic outflow values and outflow values calculated from the 0 - to 4-minute period of the suction cup decay curve. $\mathrm{C}_{\mathbf{s}}$ values were generally lower than $C_{t}$ values. Possible reasons for this difference are difficulty in measuring the first post-suction pressure and interference with aqueous formation, or drainage by the suction cup technique.

The percentage pressure decay (P.P.D.) and tonographic $\mathrm{C}$ appeared to be equally effective in separating glaucomatous from non-glaucomatous eyes.

The possible effects of the suction cup technique on aqueous humour formation and on the outflow channels are discussed. The induced rise of intra-ocular pressure or local interference with blood flow could lead to suppression of aqueous formation, and it is suggested that the restoration of the drainage channels to their true capacity may be delayed.

Practical difficulties in the use of this technique are discussed. No harmful effects were encountered. The test requires more time than tonography.

The qualitative features of the decay curve appear to be of some use in separating eyes with impaired outflow from those with normal outflow.

I wish to thank Prof. E. S. Perkins for his interest and for providing facilities in his department, and Dr. J. Gloster for his encouragement and many helpful discussions. My special thanks are due to Miss $\mathbf{M}$. Huson, who performed the tonographies, Messrs. Keeler for providing the suction cup, the Medical Illustration Department for the illustrations, and Mrs. B. Davey for her secretarial assistance.

ARmaly, M. F. (1964). Invest. Ophthal., 3, 77.

\section{REFERENCES}

Ashton, N., and Smith, R. (1953). Brit. J. Ophthal., 37, 577.

ChANDleR, M. R. (1964). Ibid., 48, 423.

Ericson, L. A. (1958). Acta ophthal. (Kbh.), suppl. 50.

Galin, M. A., Baras, I., and Mclean, J. M. (1963). Amer. J. Ophthal., 56, 883. and NANO, H. D. (1962). Ibid., 53, 927.

T,, and CAVERO, R. (1963). Arch. Ophthal., 70, 202.

LANGHAM, M. E. (1962). Invest. Ophthal., 1, 484. (1963). Exp. Eye Res., 2, 314.

— and Maumene, A. E. (1964). Trans. Amer. Acad. Ophthal., 68, 277.

Rosengren, B. (1956). Trans. ophthal. Soc. U.K., 76, 65. 